\title{
Malperformance in Verbal Fluency and Delayed Recall as Cognitive Risk Factors for Impairment in Instrumental Activities of Daily Living
}

\author{
Mirjam Koehler ${ }^{\mathrm{a}}$ Matthias Kliegel $^{\mathrm{c}}$ Birgitt Wiese $^{\mathrm{d}}$ Horst Bickel $^{\mathrm{e}}$ Hanna Kaduszkiewicz $^{\mathrm{a}}$ \\ Hendrik van den Bussche ${ }^{a}$ Sandra Eifflaender-Gorfer ${ }^{f}$ Marion Eisele ${ }^{a}$ Angela Fuchs ${ }^{g}$ \\ Hans-Helmut Koenig $^{\mathrm{b}}$ Hanna Leicht ${ }^{\mathrm{b}}$ Tobias Luck ${ }^{\mathrm{h}}$ Wolfgang Maier ${ }^{\mathrm{i}}$ Edelgard Moesch ${ }^{\mathrm{e}}$ \\ Steffi Riedel-Heller ${ }^{h}$ Franziska Tebarth ${ }^{i}$ Michael Wagner ${ }^{i}$ Siegfried Weyerer ${ }^{f}$ \\ Thomas Zimmermann ${ }^{a}$ Michael Pentzek ${ }^{g}$ for the AgeCoDe study group \\ Departments of a Primary Medical Care and ${ }^{\mathrm{b}}$ Medical Sociology and Health Economics, University Medical \\ Center Hamburg-Eppendorf, Hamburg, ' Department of Psychology, Dresden University of Technology, Dresden, \\ ${ }^{d}$ Institute of Biometrics, Hanover Medical School, Hanover, ${ }^{e}$ Department of Psychiatry, Technical University of \\ Munich, Munich, ${ }^{\mathrm{f} C e n t r a l}$ Institute of Mental Health, Mannheim, ${ }^{9}$ University Hospital Düsseldorf, Department of \\ General Practice, Heinrich Heine University Düsseldorf, Düsseldorf, h' Department of Psychiatry, University of \\ Leipzig, Leipzig, and 'Department of Psychiatry, University of Bonn, Bonn, Germany
}

\section{Key Words}

Cognition • Executive functioning • Episodic memory • Instrumental activities of daily living $\cdot$ Neuropsychological tests $\cdot$ Geriatric assessment $\cdot$ Primary health care

\begin{abstract}
Background: Maintaining independence in instrumental activities of daily living (IADL) is crucial for older adults. This study explored the association between cognitive and functional performance in general and in single IADL domains. Also, risk factors for developing IADL impairment were assessed. Methods: Here, 3,215 patients aged 75-98 years were included. Data were collected during home visits. $\boldsymbol{R} \boldsymbol{e}$ sults: Cognitive functioning was associated with IADL both cross-sectionally and longitudinally. Regarding the single IADL domains cross-sectionally, executive functioning was especially associated with shopping, while episodic memory was associated with responsibility for own medication. Conclusion: Reduced performance in neuropsychological tests is associated with a greater risk of current and subsequent functional impairment.

Copyright $\odot 2011$ S. Karger AG, Basel
\end{abstract}

\section{Introduction}

Living independently as long as possible is considered as one of the main objectives of community-dwelling older adults [1] and has a relevant impact on their quality of life [2]. In general, 2 essential types of activities in everyday life are distinguished that determine the level of autonomy and independence: basic activities of daily living (ADL) and instrumental activities of daily living (IADL).

While ADL comprise basic daily routines like bathing, eating or dressing, IADL play an important role in handling more complex activities like shopping, managing finances and preparing meals [3]. Therefore, most

Further members of the AgeCoDe study group: Heinz-Harald Abholz, Cadja Bachmann, Wolfgang Blank, Melanie Daerr, Moritz Daerr, Sven Heinrich, Frank Jessen, Teresa Kaufeler, Melanie Luppa, Manfred Mayer, Julia Olbrich, Sandra Schmitz, Britta Schürmann, Janine Stein, Kristina Stock, Klaus Weckbecker, Dagmar Weeg, Jochen Werle and Anja Wollny. Principal investigators: Wolfgang Maier and Hendrik van den Bussche.

\section{KARGER}

Fax +41613061234 E-Mail karger@karger.ch www.karger.com

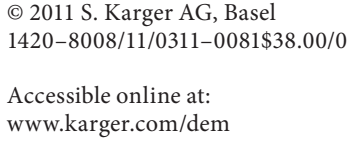


IADL instruments focus on complex everyday living skills [4-9].

Impairment in IADL usually precedes ADL impairment [10] and is thought to be particularly associated with cognitive functioning. Moreover, it has been argued that specific cognitive measures are more appropriate for establishing an association between cognition and IADL than global cognitive status measures [6]. In a cross-sectional study, executive functioning (EF) was detected to be a more powerful predictor of IADL than other cognitive constructs including memory, visuospatial functioning or motor functioning [11]. Recently, initial longitudinal evidence has been reported in a Japanese sample that examined the association between specific cognitive variables and functional decline and found processing speed and EF to be associated with IADL [7]. Moreover, another study [12] revealed a unique association between EF and IADL longitudinally, while in cross-sectional analyses both a composite memory score and a composite EF score were associated with an approximation of IADL functioning. Tomaszewski et al. [13] analyzed participants of the same longitudinal study concerning the relationship between decline in the IADL measure applied and decline in memory and EF. They detected an association between both cognitive constructs and instrumental activities. In contrast, Royall et al. [14] found an association between declining EF and performance of IADL, whereas changes in a verbal memory test were not associated with change in IADL [15]. Taken together, it is still unclear whether only EF or both EF and memory are associated with and predict IADL impairment. Another important question is whether the association between cognition and IADL is the same for all IADL items or more pronounced for some activities than for others. Studies suggest that cognitive performance is especially associated with managing finances, responsibility for medication intake and telephone use [6, 16-18]. However, so far, no systematic comparison between EF and memory and their associations with a set of single IADL has been reported.

Hence, the aim of this study was first to determine whether EF and/or episodic memory are associated with an older person's ability to perform IADL and whether they can predict decline on IADL. The second aim was to study the association between EF and episodic memory with specific IADL items in order to identify those items being more associated with EF and/or episodic memory.

We hypothesized that in cross-sectional analyses, both episodic memory and EF will be associated with
IADL in general. However, as specific IADL items involve different cognitive abilities, we predicted EF to be associated with telephone use, managing finances and responsibility for medication intake as those activities require planning and sequencing of activities. On the other hand, we assumed memory to be related to activities which also require episodic memory like responsibility for medication intake and shopping. Concerning longitudinal analyses, we hypothesized, in line with prior results $[7,12]$, that EF, but not episodic memory, is associated with IADL impairment.

\section{Materials and Methods}

\section{Design}

This study is part of the prospective longitudinal multicenter German study on Ageing, Cognition and Dementia in Primary Care Patients (AgeCoDe) with data collection between January 2003 and December 2007. The study was completed in accordance with the Helsinki declaration and approved by the local ethics committee. All participants gave written informed consent prior to study entry.

\section{Participants}

General practitioners (GPs) in 6 German cities (Bonn, Düsseldorf, Hamburg, Leipzig, Mannheim and Munich) were asked to take part in this study. In total, 138 GPs participated in the recruitment process, ranging from 19 to 29 GPs per study center. Inclusion criteria for patients were age 75 years and older, absence of dementia and at least 1 contact with the GP within the last 12 months. Exclusion criteria were insufficient German language skills, consultations only by home visits or not being a regular patient of the participating GP, residence in a nursing home, severe illness the GP would deem fatal within 3 months, deafness or blindness and lacking ability to consent. On average, each GP included 24 randomly selected patients.

As shown in figure 1, 3,327 GP patients participated in the baseline assessment. From this sample, 2,820 patients still participated in follow-up 1 (FU1) 18 months after the baseline assessment and 2,478 patients in follow-up 2 (FU2) 36 months after the baseline assessment. Out of these 2,478 patients, 18 patients attended FU2, but not FU1. Thus, in total 2,460 patients were assessed 3 times.

Out of the total baseline sample, 112 patients (3.4\%) had to be excluded in the following analyses due to their age being below 75 years $(39,1.2 \%)$, due to being classified as having dementia after baseline neuropsychological testing $(70,2.1 \%)$ or due to incomplete neuropsychological assessments $(3,0.1 \%)$. Therefore, a total of 3,215 patients were included for analyses at baseline, 2,741 for FU1 and 2,411 for FU2.

Comparing participants with nonparticipants at baseline, earlier publications of the AgeCoDe study group $[19,20]$ demonstrated that patients refusing participation were significantly older and included fewer males when compared to participating patients. A comparison of dropouts and study participants remaining in the sample till follow-up assessment revealed that, regard- 


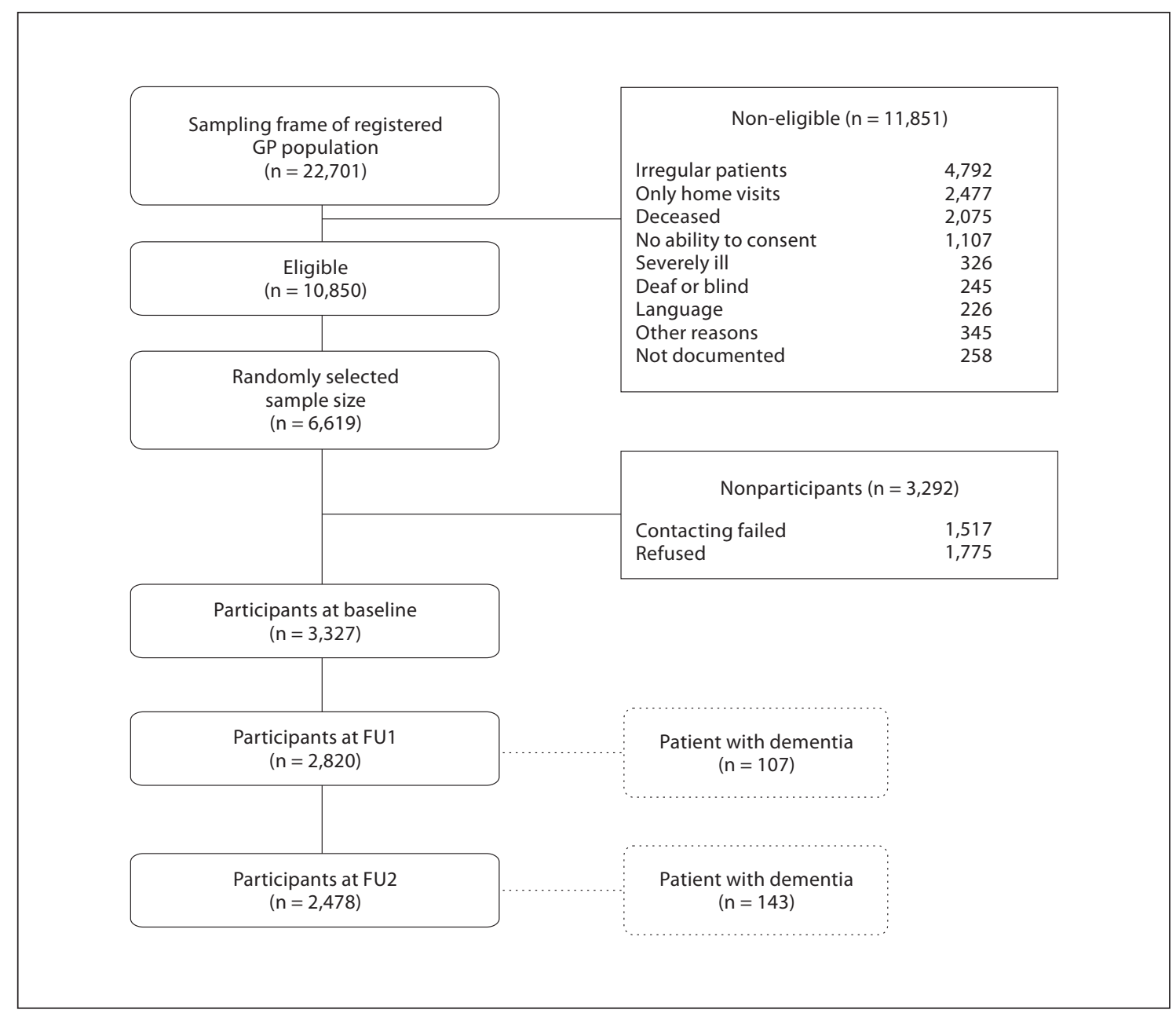

Fig. 1. Sample attrition and sample.

ing baseline data, dropouts in FU1 and FU2 were significantly older and had a lower MMSE score [FU1: $\mathrm{t}(658)=3.635, \mathrm{p}<0.01$ for age, $\mathrm{t}(607)=-9.137, \mathrm{p}<0.01$ for MMSE; FU2: $\mathrm{t}(448)=5.106$, $\mathrm{p}<0.01$ for age, $\mathrm{t}(453)=-6.239, \mathrm{p}<0.01$ for MMSE]. Hence, in those variables, a bias has to be assumed, although the differences were small. No difference was found regarding males and females participating in follow-up assessments compared with those who had left the study $\left(\chi^{2}=0.56\right.$, d.f. $=1, p=0.45$ for FU1 and $\chi^{2}=3.70$, d.f. $=1, p=0.06$ for FU2).

\section{Instruments and Data Collection}

Structured clinical interviews were carried out at the patients' home by trained physicians and psychologists. All interviews included neuropsychological testing, collection of sociodemographic data, evaluation of functional activities, examination of current medications, suspected risk factors for dementia, and screening for depressive symptoms. For the latter, a German version of the 15-item Geriatric Depression Scale (GDS) [21] was applied. Possible impairment in mobility, vision and hearing was assessed by self- or informant-based rating on a 4-grade scale (no, slight, serious or severe impairment).

Neuropsychological assessment consisted of the Structured Interview for Diagnosis of Dementia of Alzheimer type, Multiinfarct Dementia and Dementia of other Etiology according to DSM-III-R, DSM-IV and ICD-10 (SIDAM) [22]. The SIDAM is a neuropsychological test battery including 55 cognitive test items and a section for clinical examination and diagnosis. Dementia cases were diagnosed according to DSM-IV criteria [23] following the diagnostic algorithm of the SIDAM and the Global Deterioration Scale [24] which had to be scored with at least 4. Unclear cases were discussed in a standing diagnostic clearing platform with multiple experts in the field. Furthermore, subtests of the neuropsychological test battery of the Consortium to Establish a Registry for Alzheimer's Disease (CERAD-NP) [25] were administered: verbal fluency, word list learning and recall, word list delayed recall, word list recognition.

We assessed EF using the CERAD semantic verbal fluency scale [26]. In this scale, participants have to name as many ani- 
Table 1. Means and standard deviations of verbal fluency, delayed recall and GDS score

\begin{tabular}{|c|c|c|c|c|c|c|}
\hline \multirow[t]{2}{*}{ Measures } & \multicolumn{2}{|c|}{ Baseline } & \multicolumn{2}{|l|}{ FU1 } & \multicolumn{2}{|l|}{ FU2 } \\
\hline & $\mathrm{n}^{\mathrm{a}}$ & mean $(\mathrm{SD})$ & $\mathrm{n}^{\mathrm{a}}$ & mean $(\mathrm{SD})$ & $\mathrm{n}^{\mathrm{a}}$ & mean $(\mathrm{SD})$ \\
\hline Verbal fluency & 3,214 & $19.47(5.44)$ & 2,730 & $19.61(5.86)$ & 2,377 & $19.67(6.27)$ \\
\hline Delayed recall & 3,191 & $5.43(2.22)$ & 2,701 & $5.57(2.40)$ & 2,338 & $5.69(2.63)$ \\
\hline GDS score & 3,211 & $2.25(2.31)$ & 2,727 & $2.46(2.43)$ & 2,376 & $2.53(2.49)$ \\
\hline
\end{tabular}

${ }^{\text {a }}$ Variable $\mathrm{n}$ due to missing data. mals as possible during a 1-min time period. The higher the verbal fluency score which indicates more generated words, the better the performance. To assess episodic memory, the amount of words recalled after $10 \mathrm{~min}$ in the delayed recall task of the CERAD with a range between 0 and 10 recalled items was computed.

The functional status of the patients was assessed by the IADL scale [3]. This IADL scale (Cronbach's alpha in the current sample $=0.66)$ consists of 8 items describing the patients' abilities in the following dimensions: ability to use telephone, shopping, food preparation, housekeeping, laundry, mode of transportation, responsibility for own medications and the ability to handle finances. Each item allowed a rating of at least 3 grades (independent management, some assistance required, completely dependent) at which possible confounding factors (physical impairments) were excluded. Could a patient, for example, not leave his or her house due to hindered mobility but was able to write a shopping list independently, the item shopping was scored as 'takes care of all shopping needs independently'. Impairment was true in case the patient required help or was completely unable to perform the dimension independently. For each item, the participant could achieve either 0 or 1 point as defined in the original scale [3]. As some items did not apply to all participants (e.g. food preparation, housekeeping and laundry for men whose wives fulfilled these tasks), their answers were recoded for analysis as having no difficulty in performing the task following the approach of prior publications $[6,27]$.

\section{Statistical Analysis}

First, logistic regression analyses using SPSS version 16.0 were conducted to identify the amount of explained variance in IADL by EF and episodic memory cross-sectionally [28]. For the logistic regressions, the IADL scale was recoded in a dichotomous variable with IADL impairment assumed when at least 1 of the 8 abilities was impaired and thus scored with 0 (for a similar approach, see Jefferson et al. [27]). Additional logistic regression analyses were then realized for all single IADL items which were also scored as either impaired or not impaired. Thus, IADL in general or single IADL items always served as dependent variable. EF and episodic memory were the independent variables of interest. All analyses were controlled for age, gender, education, impairment in mobility, vision and hearing as well as depressive symptoms.

To discover changes over a 3-year period, Cox regressions were carried out with IADL impairment as event to occur. In order to avoid an overestimation of time to IADL impairment, months till impairment were assumed as half of the time till the next observation. The whole time interval was taken into account when no functional impairment occurred. In all analyses, a p value less than 0.05 was considered statistically significant.

\section{Results}

\section{Patients}

A total of 3,215 participants on average aged 79.7 years (SD 3.6) were used for all subsequent analyses, including 2,104 women (65\%) and 1,111 men (35\%). In FU1, 2,741 participants with an average age of 81.2 years (SD 3.5) remained in the sample comprising 1,785 (65\%) women and 956 (35\%) men. Finally, out of the 2,411 participants in FU2, 1,590 were female (66\%) and 821 were male (34\%). The mean age here was 82.5 years (SD 3.4).

Regarding IADL impairment, 241 patients (7.5\%) were impaired at baseline assessment, 237 (8.7\%) at FU1 and 234 (9.7\%) at FU2. For means and standard deviations of verbal fluency, delayed recall and the GDS, see table 1.

\section{Outcomes}

The cross-sectional analysis using logistic regression revealed an association between cognition and IADL impairment after controlling for age, gender, education, impairment in mobility, vision or hearing and depression (for odds ratios and confidence intervals, see table 2). Episodic memory (measured by delayed recall) showed a somewhat higher association with IADL impairment than EF (measured by verbal fluency). Higher age, male gender, impaired mobility and a higher score on the GDS were also associated with IADL impairment.

Analyzing the IADL domains at baseline separately, IADL impairment was most frequent in shopping $(\mathrm{n}=$ 116), followed by responsibility for own medication ( $\mathrm{n}=$ 93), food preparation ( $n=63)$, laundry $(n=39)$ and transportation $(n=35)$. Housekeeping $(n=12)$, handling finances $(n=10)$ as well as using the telephone 
Table 2. Cross-sectional associations between EF (verbal fluency) and episodic memory (delayed recall) with IADL impairment (results of logistic regression at baseline)

\begin{tabular}{lllll}
\hline Baseline characteristics & $\mathrm{n}$ & $\begin{array}{l}\text { Odds } \\
\text { ratio }\end{array}$ & $95 \% \mathrm{CI}$ & $\mathrm{p}$ \\
\hline Verbal fluency (uncorr) & 3,187 & 0.924 & $0.900-0.949$ & $<0.001$ \\
Delayed recall (uncorr) & 3,187 & 0.816 & $0.769-0.867$ & $<0.001$ \\
Verbal fluency (corr) & 3,187 & 0.969 & $0.940-0.998$ & $<0.05$ \\
Delayed recall (corr) & 3,187 & 0.906 & $0.844-0.972$ & $<0.01$ \\
\hline
\end{tabular}

All odds ratios are referred to IADL impairment. 237 patients were regarded as IADL impaired (= impaired in at least $1 \mathrm{IADL})$. uncorr $=$ Univariable results; corr $=$ corrected for age, gender, education, mobility, vision, hearing, and GDS score, $\mathrm{R}^{2}=0.20$.

Table 3. Risk of IADL impairment as event to occur within 3 years (results of Cox regression model)

\begin{tabular}{|c|c|c|c|c|c|c|c|c|}
\hline \multirow[t]{2}{*}{ Baseline characteristics } & \multicolumn{4}{|c|}{ With demented patients at FU1 and FU2 } & \multicolumn{4}{|c|}{ Without demented patients } \\
\hline & $\mathrm{n}$ & hazard ratio & $95 \% \mathrm{CI}$ & $\mathrm{p}$ & $\mathrm{n}$ & hazard ratio & $95 \% \mathrm{CI}$ & $\mathrm{p}$ \\
\hline Verbal fluency (uncorr) & 2,564 & 0.891 & $0.870-0.913$ & $<0.001$ & 2,432 & 0.925 & $0.898-0.952$ & $<0.001$ \\
\hline Delayed recall (uncorr) & 2,551 & 0.723 & $0.685-0.763$ & $<0.001$ & 2,423 & 0.787 & $0.736-0.843$ & $<0.001$ \\
\hline Verbal fluency (corr) & 2,547 & 0.937 & $0.913-0.962$ & $<0.001$ & 2,421 & 0.955 & $0.926-0.986$ & $<0.01$ \\
\hline Delayed recall (corr) & 2,547 & 0.802 & $0.757-0.850$ & $<0.001$ & 2,421 & 0.855 & $0.795-0.920$ & $<0.001$ \\
\hline
\end{tabular}

All hazard ratios are referred to IADL impairment. uncorr = Univariable results; corr = corrected for age, gender, education, mobility, vision, hearing, and GDS score.

$(\mathrm{n}=2)$ were less frequently affected. In separate logistic regressions, an association between $\mathrm{EF}$ and single IADL was observed for shopping $(\mathrm{OR}=0.943,95 \% \mathrm{CI}=$ $0.904-0.984, p<0.01$ ), responsibility for own medication $(\mathrm{OR}=0.949,95 \% \mathrm{CI}=0.907-994, \mathrm{p}<0.05)$ and finances $(\mathrm{OR}=0.821,95 \% \mathrm{CI}=0.681-0.990, \mathrm{p}<0.05)$. Episodic memory was only associated with responsibility for own medication (OR $=0.828,95 \%$ CI $=0.742-$ $0.924, \mathrm{p}<0.01)$. In order to correct for multiple testing, we used the Holm-Bonferroni method [29]. This indicated that the association between $\mathrm{EF}$ and responsibility for own medication as well as the association between $\mathrm{EF}$ and finances might be false-positive results. Hence, the association between EF and shopping and the association between episodic memory and responsibility for own medication emerged as reliable findings even when adjusting for multiple testing.

Utilizing a longitudinal approach, a Cox regression model using the same covariates as mentioned above, revealed that worse performance in EF and episodic memory indicated a greater potential for IADL impairment to occur (table 3). Specifically, worse performance in episodic memory was slightly more associated with IADL impairment than worse performance in EF. This pattern persisted after exclusion of demented persons from FU1 and FU2. Besides the 2 cognitive measures, higher age, impairment in mobility and hearing (hearing only for the calculation including demented patients) and a higher GDS score were significantly associated with a higher risk of IADL impairment in the course of 3 years.

\section{Discussion}

The present study clearly reveals that results in neuropsychological tests are associated with the functional status of elderly GP patients. Performing worse on neuropsychological tests including verbal fluency and delayed recall of words increases the risk of functional impairment. This is true both for cross-sectional and longitudinal analyses.

Cross-sectional results here fit in nicely with previous results suggesting that low performance in EF is associ- 
ated with IADL impairment $[11,30]$. However, the episodic memory task in our study demonstrated a somewhat higher explanatory value than $\mathrm{EF}$, which is in contrast to the results of Cahn-Weiner et al. [11]. An important novel finding concerns the disentangling of single IADL. Considering the IADL domains separately, former results indicating that responsibility for medication intake is associated with EF and memory could be confirmed [17]. However, the association between EF and medication intake might be a false-positive result according to the Holm-Bonferroni method. All other IADL domains showed no significant association with episodic memory while EF was also associated with shopping and possibly with finances. This is in line with those tasks being considered as complex activities with a need for planning and sequencing. Surprisingly, telephone use was not associated with EF or episodic memory, which is likely to be an artifact due to the very small sample size of only 2 impaired participants.

Longitudinal analyses corroborated cross-sectional findings and again demonstrated an association between cognition and IADL. IADL impairment was slightly less likely to occur when the patients performed better in the episodic memory task compared with performance in the EF task. This is in contrast to prior results showing that EF becomes more important regarding the temporal course of functional impairment [12] and IADL decline [7], but in line with previous results showing that memory and EF are associated with IADL [31].

The present data set also allowed for an important methodological clarification that critically extends some previous studies: noteworthy, the significant association between cognition and IADL is longitudinally not just an effect of increasing functional impairment due to dementia. After excluding incident dementia cases, the association slightly diminished, but still remained significant indicating the robustness of the effects and an independent explanatory value of episodic memory and EF. Some attention needs to be drawn to patients with mild cognitive impairment who are still included in the analyses. It is discussed that mild cognitive impairment patients may have impairments in IADL, although less frequently than patients with dementia [32]. However, additional analyses (data not shown) excluding mild cognitive impairment did not change the results.

There are several strengths and limitations of this study. First, our multicenter study was specifically designed to investigate older GP patients. Thus our study population can be assumed to be representative of older adults. Furthermore, most studies on the relationship between cognitive functioning and IADL impairment have focused on persons under the age of 75 . To study a large sample of elderly persons above 75 years is particularly important since cognitive and functional impairment become more common with increasing age. Second, as stated by Barberger-Gateau and Fabrigoule [33], 'cross-sectional studies tend to overestimate differences in cognitive functioning with age', so we also used a longitudinal design with standardized time intervals. However, as reported above, it can be assumed that participants lost to follow-up assessments were somewhat older and more vulnerable to cognitive impairment resulting possibly in an underestimation of effects in the remaining sample. Yet, this limitation applies to most longitudinal studies [34, 35]. Third, neuropsychological assessments and the evaluations of the person's actual life circumstances including the ability to perform IADL independently can be regarded as highly reliable due to home visits by trained physicians and psychologists and additional interviews with close relatives or friends if cognitive function was impaired. Additionally, according to Crawford and Henry [26], verbal fluency tasks are a powerful EF measure with acceptable reliability and validity, short test duration and are easy to interpret. Thus, even if one can consider this as a narrow approach to studying EF, verbal fluency tests are specific and sensitive to executive dysfunction $[36,37]$. However, we want to add as limitation that we cannot fully preclude that exactly the same results would occur when measuring EF and episodic memory with other cognitive tests than presented here. Finally, the present study applied an IADL measurement focusing on items of IADL performance like performing household tasks or managing money in contrast to broader and more unspecific IADL measurements used earlier such as the Blessed Roth Dementia Rating Scale $[12,13]$.

In conclusion, results here suggest that higher-order cognitive functioning can be important in predicting the progression to IADL impairment in older adults. Furthermore, besides decline in episodic memory, impairment in EF was found to be an important predictor of restricted autonomy. Thus, episodic memory and EF should be assessed carefully to detect impending IADL impairment, especially in responsibility for own medication and shopping. 


\section{Acknowledgements}

We want to thank all participating patients and their general practitioners for their good collaboration. This publication is part of the German Research Network on Dementia (KND) and the German Research Network on Degenerative Dementia (KNDD) and was funded by the German Federal Ministry of Education and Research (grants KND: 01GI0102, 01GI0420, 01GI0422,
01GI0423, 01GI0429, 01GI0431, 01GI0433, 01GI0434; grants KNDD: O1GI0710, 01GI0711, 01GI0712, 01GI0713, 01GI0714, 01GI0715, 01GI0716)

\section{Disclosure Statement}

No potential conflicts.

\section{References}

1 Stuck AE, Walthert JM, Nikolaus T, Büla CJ, Hohmann C, Beck JC: Risk factors for functional status decline in community-living elderly people: a systematic literature review. Soc Sci Med 1999;48:445-469.

2 Kresevic DM: Assessment of function; in Capezuti EA, Zwicker D, Mezey M (eds): Evidence-Based Geriatric Nursing Protocols for Best Practice, ed 3. New York, Springer, 2008, pp 23-40.

3 Lawton MP, Brody EM: Assessment of older people: self-maintaining and instrumental activities of daily living. Gerontologist 1969; 9:179-186.

4 Bell-McGinty S, Podell K, Franzen M, Baird AD, Williams MJ: Standard measures of executive function in predicting instrumental activities of daily living in older adults. Int J Geriatr Psychiatry 2002;17:828-834.

5 Burton CL, Strauss E, Hultsch DF, Hunter MA: Cognitive functioning and everyday problem solving in older adults. Clin Neuropsychol 2006;20:432-452.

6 Grigsby J, Kaye K, Baxter J, Shetterly SM, Hamman RF: Executive cognitive abilities and functional status among communitydwelling older persons in the San Luis Valley Health and Aging Study. J Am Geriatr Soc 1998:46:590-596.

7 Iwasa H, Gondo Y, Yoshida Y, Kwon J, Inagaki H, Kawaai C, Masui Y, Kim H, Yoshida $\mathrm{H}$, Suzuki T: Cognitive performance as a predictor of functional decline among the nondisabled elderly dwelling in a Japanese community: a 4-year population-based prospective cohort study. Arch Gerontol Geriatr 2008;47:139-149.

8 Jefferson AL, Byerly LK, Vanderhill S, Lambe S, Wong S, Ozonoff A, Karlawish JH: Characterization of activities of daily living in individuals with mild cognitive impairment. Am J Geriatr Psychiatry 2008; 16:375383.

9 Lewis MS, Miller LS: Executive control functioning and functional ability in older adults. Clin Neuropsychol 2007;21:274-285.

10 Graf C: The Lawton instrumental activities of daily living scale. Am J Nurs 2008;108:5262.

11 Cahn-Weiner DA, Malloy PF, Boyle PA, Marran M, Salloway S: Prediction of func- tional status from neuropsychological tests in community-dwelling elderly individuals. Clin Neuropsychol 2000;14:187-195.

12 Cahn-Weiner DA, Tomaszewski Farias S, Julian L, Harvey DJ, Kramer JH, Reed BR, Mungas D, Wetzel M, Chui H: Cognitive and neuroimaging predictors of instrumental activities of daily living. J Int Neuropsychol Soc 2007;13:747-757.

13 Tomaszewski S, Cahn-Weiner DA, Harvey DJ, Reed BR, Mungas D, Kramer JH, Chui H: Longitudinal changes in memory and executive functioning are associated with longitudinal change in instrumental activities of daily living in older adults. Clin Neuropsychol 2009;23:446-461.

14 Royall DR, Palmer R, Chiodo LK, Polk MJ: Declining executive control in normal aging predicts change in functional status: the Freedom House Study. J Am Geriatr Soc 2004;52:346-352.

15 Royall DR, Palmer R, Chiodo LK, Polk MJ: Executive control mediates memory's association with change in instrumental activities of daily living: the Freedom House Study. J Am Geriatr Soc 2005;53:11-17.

16 Barberger-Gateau P, Commenges D, Gagnon M, Letenneur L, et al: Instrumental activities of daily living as a screening tool for cognitive impairment and dementia in elderly community dwellers. J Am Geriatr Soc 1992; 40:1129-1134.

17 Insel K, Morrow D, Brewer B, Figueredo A: Executive function, working memory, and medication adherence among older adults. J Gerontol B Psychol Sci Soc Sci 2006;61:P102P107.

18 McGuire LC, Ford ES, Ajani UA: Cognitive functioning as a predictor of functional disability in later life. Am J Geriatr Psychiatry 2006; 14:36-42.

19 Luck T, Riedel-Heller SG, Kaduszkiewicz H, Bickel H, Jessen F, Pentzek M, Wiese B, Koelsch H, van den Bussche H, Abholz HH, Moesch E, Gorfer S, Angermeyer MC, Maier W, Weyerer S: Mild cognitive impairment in general practice: age-specific prevalence and correlate results from the German study on ageing, cognition and dementia in primary care patients (AgeCoDe). Dement Geriatr Cogn Disord 2007;24:307-316.
20 Weyerer S, Eifflaender-Gorfer S, Kohler L, Jessen F, Maier W, Fuchs A, Pentzek M, Kaduszkiewicz H, Bachmann C, Angermeyer MC, Luppa M, Wiese B, Mosch E, Bickel H, German AgeCoDe Study group: Prevalence and risk factors for depression in non-demented primary care attenders aged 75 years and older. J Affect Disord 2008;111: 153-163.

21 Sheikh JI, Yesavage JA: Geriatric depression scale (GDS): recent evidence and development of a shorter version. Clin Gerontol 1986;5:165-173.

22 Zaudig M, Hiller W: Sidam-Handbuch. Strukturiertes Interview für die Diagnose einer Demenz vom Alzheimer Typ, der Multi-Infarkt- (oder vaskulären) Demenzen und Demenzen anderer Ätiologien nach DSM-III-R, DSM-IV und ICD-10. Bern, Huber, 1996.

23 Sass H, Wittchen HU, Zaudig M, Houben I: Diagnostisches und statistisches Manual psychischer Störungen - Textrevision. Göttingen, Hogrefe, 2003.

24 Reisberg B, Ferris SH, de Leon MJ, Crook T: The global deterioration scale for assessment of primary degenerative dementia. Am J Psychiatry 1982;139:1136-1139.

25 Morris JC, Heyman A, Mohs RC, Hughes JP, van Belle G, Fillenbaum G, Mellits ED, Clark C: The Consortium to Establish a Registry for Alzheimer's Disease (CERAD). 1. Clini$\mathrm{cal}$ and neuropsychological assessment of Alzheimer's disease. Neurology 1989;39: 1159-1165.

26 Crawford JR, Henry JD: Assessment of executive deficits; in Halligan PW, Wade N (eds): The Effectiveness of Rehabilitation for Cognitive Deficits. London, Oxford University Press, 2005, pp 233-246.

27 Jefferson AL, Paul RH, Ozonoff A, Cohen RA: Evaluating elements of executive functioning as predictors of instrumental activities of daily living (IADLS). Arch Clin Neuropsychol 2006;21:311-320.

28 Schendera CF: Regressionsanalyse mit SPSS. München, Oldenbourg Wissenschaftsverlag, 2008.

29 Holm S: A simple sequentially rejective multiple test procedure. Scand J Stat 1979;6:6570 . 
30 Carlson MC, Fried LP, Xue Q-L, BandeenRoche K, Zeger SL, Brandt J: Association between executive attention and physical functional performance in community-dwelling older women. J Gerontol B Psychol Sci Soc Sci 1999;54:S262-S270.

31 Dodge HH, Du YC, Saxton JA, Ganguli M: Cognitive domains and trajectories of functional independence in nondemented elderly persons. J Gerontol A Biol Sci Med Sci 2006; 61:1330-1337.
32 Pérès K, Chrysostome V, Fabrigoule C, Orgogozo JM, Dartigues JF, Barberger-Gateau $\mathrm{P}$ : Restriction in complex activities of daily living in MCI. Neurology 2006;67:461-466.

33 Barberger-Gateau P, Fabrigoule C: Disability and cognitive impairment in the elderly. Disabil Rehabil 1997;19:175-193.

34 Carlson MC, Xue QL, Zhou J, Fried LP, Carlson MC, Xue Q-L, Zhou J, Fried LP: Executive decline and dysfunction precedes declines in memory: the Women's Health and Aging Study II. J Gerontol A Biol Sci Med Sci 2009;64:110-117.
35 Singer T, Verhaeghen P, Ghisletta P, Lindenberger U, Baltes PB: The fate of cognition in very old age: six-year longitudinal findings in the Berlin Aging Study (BASE). Psychol Aging 2003;18:318-331.

36 Jurado MB, Rosselli M: The elusive nature of executive functions: a review of our current understanding. Neuropsychol Rev 2007;17: 213-233.

37 Kennedy GJ, Smyth CA: Screening older adults for executive dysfunction. Am J Nurs 2008;108:62-71. 